\title{
A unique profile of serum cytokines in type 1 autoimmune pancreatitis and chronic rhinosinusitis
}

\author{
Tomoe Yoshikawa, ${ }_{1}$ Kosuke Minaga, ${ }^{1}$ Akane Hara, ${ }^{1}$ Ikue Sekai, ${ }^{1}$ Yasuo Otsuka, ${ }^{1}$ Ryutaro Takada, ${ }^{1}$ \\ Ken Kamata, ${ }^{1}$ Tomohiro Watanabe, ${ }^{1}$ Masatoshi Kudo ${ }^{1}$
}

\begin{abstract}
Background: Type 1 autoimmune pancreatitis (AIP) is a pancreatic manifestation of IgG4-related disease (IgG4-RD). Although AIP and IgG4-RD are characterized by multiple organ involvement including salivary glands, lung, and kidney, co-occurrence of chronic rhinosinusitis (CRS) and AIP/IgG4-RD has been poorly defined.

Objective: We explored molecular mechanism accounting for the co-occurrence of CRS and AIP/IgG4-RD.

Methods: Serum concentrations of IFN- $\alpha$ and IL-33 were measured by enzyme-linked immune-sorbent assay.

Results: We encountered a patient with concurrent type 1 AIP/IgG4-RD and CRS. Induction of remission by prednisolone (PSL) for type 1 AIP/IgG4-RD led to a marked improvement of CRS. Serum cytokine analysis after PSL treatment revealed a marked reduction in serum concentrations of IFN- $\alpha$ and IL-33, both of which are candidate pathogenic cytokines for AIP/IgG4-RD.
\end{abstract}

Conclusions: Given that IL-33 is shared as one of pathogenic cytokines by type 1 AIP/IgG4-RD and CRS, enhanced IL33 responses may cause concurrent type 1 AIP/IgG4-RD and CRS.

Key words: type 1 autoimmune pancreatitis, IgG4-related disease, chronic rhinosinusitis, steroid, cytokine, plasmacytoid dendritic cell (pDC), IL-33

From:

${ }^{1}$ Department of Gastroenterology and Hepatology, Kindai University Faculty of Medicine, Osaka-Sayama, Osaka, Japan

\section{Corresponding author:}

Kosuke Minaga

Department of Gastroenterology and Hepatology, Kindai University Faculty of Medicine, 377-2 Ohno-Higashi, Osaka-Sayama, 589-8511 Osaka, Japan

E-mail: kousukeminaga@med.kindai.ac.jp

\section{Introduction}

Type 1 autoimmune pancreatitis (AIP) is a pancreatic manifestation of systemic IgG4-related disease (IgG4-RD)., ${ }^{1,2}$ IgG4-RD is a newly proposed disease entity characterized by elevated levels of serum IgG4 antibody (Ab) concentrations, infiltration of IgG4-expressing plasma cells into the target organs, and multiple organ involvement. ${ }^{1,2}$ Although immunopathogenesis of type 1 AIP and IgG4-RD has not been fully understood, we recently provided evidence that plasmacytoid dendritic cells (pDCs) producing both IFN- $\alpha$ and IL-33 mediate chronic fibroinflammatory responses characterizing these disorders. ${ }^{3-7}$ Indeed, serum concentrations of IFN- $\alpha$ and IL-33 were significantly higher in patients with IgG4-RD and type 1 AIP than in those with chronic alcoholic pancreatitis or healthy controls. ${ }^{8}$ Moreover, induction of remission by prednisolone (PSL) was accompanied by marked reductions in serum concentrations of IFN- $\alpha$ and IL-33 in patients with type 1 AIP and IgG4-RD. ${ }^{8,9}$ Thus, IFN- $\alpha$ and IL-33 play pathogenic roles in the development of type 1 AIP and IgG4RD.

IgG4-RD preferentially affects the pancreas, bile duct, salivary glands, lung, and kidney. ${ }^{1,2}$ It should be noted, however, that this autoimmune disorder could involve almost all of the organs. ${ }^{1,2}$ Chronic rhinosinusitis (CRS) is an inflammatory disease of the paranasal sinuses. ${ }^{10}$ Although the incidence of CRS in patients with IgG4-RD is very high $(65.2 \%),{ }^{11}$ the molecular mechanisms underlying such co-occurrence of both disorders have been poorly defined. We have encountered a case that exhibited a unique profile of serum cytokines 
in concurrent IgG4-RD and CRS. This case strongly suggests that IgG4-RD and CRS might share one pathogenic cytokine responses. The institutional review board of the Kindai University Hospital approved this study (approval number: 28034).

\section{Case Report}

A 48-year-old woman visited Kindai University Hospital (Osaka-Sayama, Japan) for annual checkup. She has had CRS for 20 years, which has not been resolved with repeated macrolide antibiotic treatment for 5 months; her past medical history included rheumatoid arthritis, and asthma. She had been diagnosed with rheumatoid arthritis 15 years ago and had been treated first with Infliximab but she is currently treated with Tocilizumab. She has had asthma for 8 years and is treated with combination of inhaled corticosteroid and a long-acting beta-2 adrenergic agonist combination when she has symptoms. On ${ }^{18} \mathrm{~F}$-fluorodeoxyglucose positron emission tomography (FDG-PET), there was remarkable uptake of FDG

\section{a}

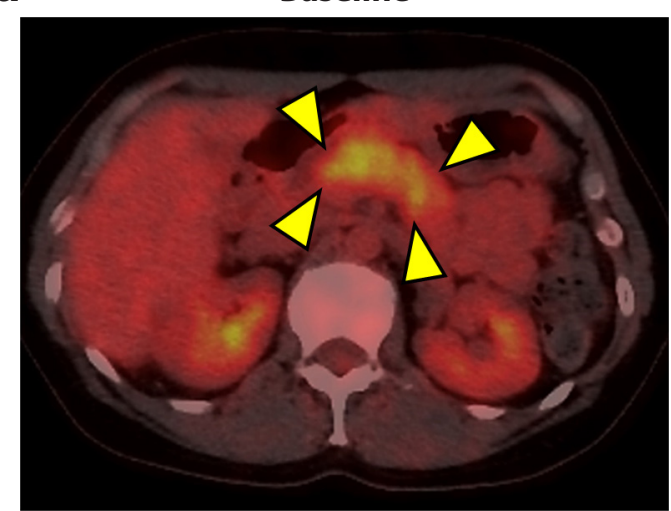

b

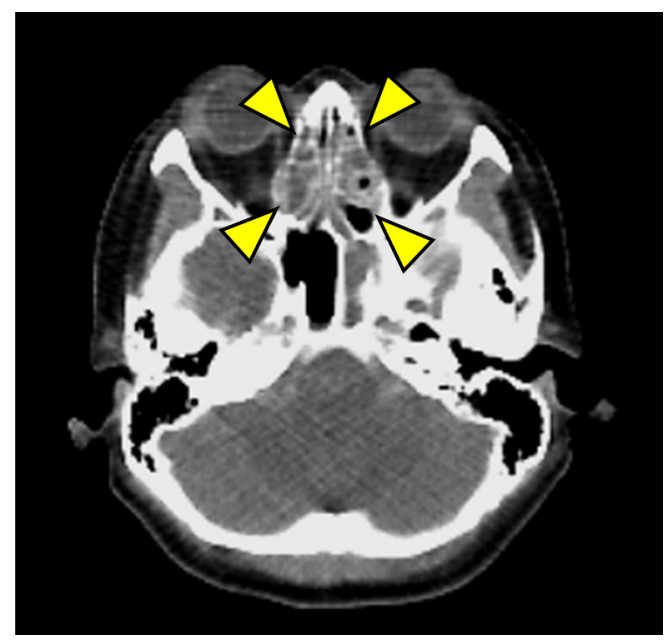

in an enlarged pancreas (Figure 1a, left), parotid, submandibular, and lacrimal glands, all of which are preferentially affected by IgG4-RD. ${ }^{1,2}$ Computed tomography (CT) showed severe mucosal hypertrophy in the bilateral ethmoid, left sphenoid, and frontal sinuses (Figure 1b, left), but there were no nasal polyps. These FDG-PET and CT findings suspected concurrent IgG4-RD and CRS. ${ }^{1,2,12}$ Her serum concentrations of IgG, IgG4, and IgE were $3075 \mathrm{mg} / \mathrm{dL}$ (normal, 861-1747 mg/ $\mathrm{dL}$ ), $2090 \mathrm{mg} / \mathrm{dL}$ (normal, 4.5-117 mg/dL), and $209 \mathrm{IU} / \mathrm{mL}$ (normal, < $170 \mathrm{IU} / \mathrm{mL}$ ), respectively. Immunological investigations such as rheumatoid factor, anti-nuclear antibody and anti-double stranded DNA antibody tests were negative. No major abnormal findings were detected in complete blood cell counts and biochemical analyses. To ascertain the diagnosis of IgG4-RD involving the pancreas and the salivary and lacrimal glands, endoscopic ultrasound-guided fine needle aspiration of the enlarged pancreas was performed. Figure 1c illustrates the formation of storiform fibrosis and prominent infiltration of IgG4-expressing plasmacytes, both of which are the
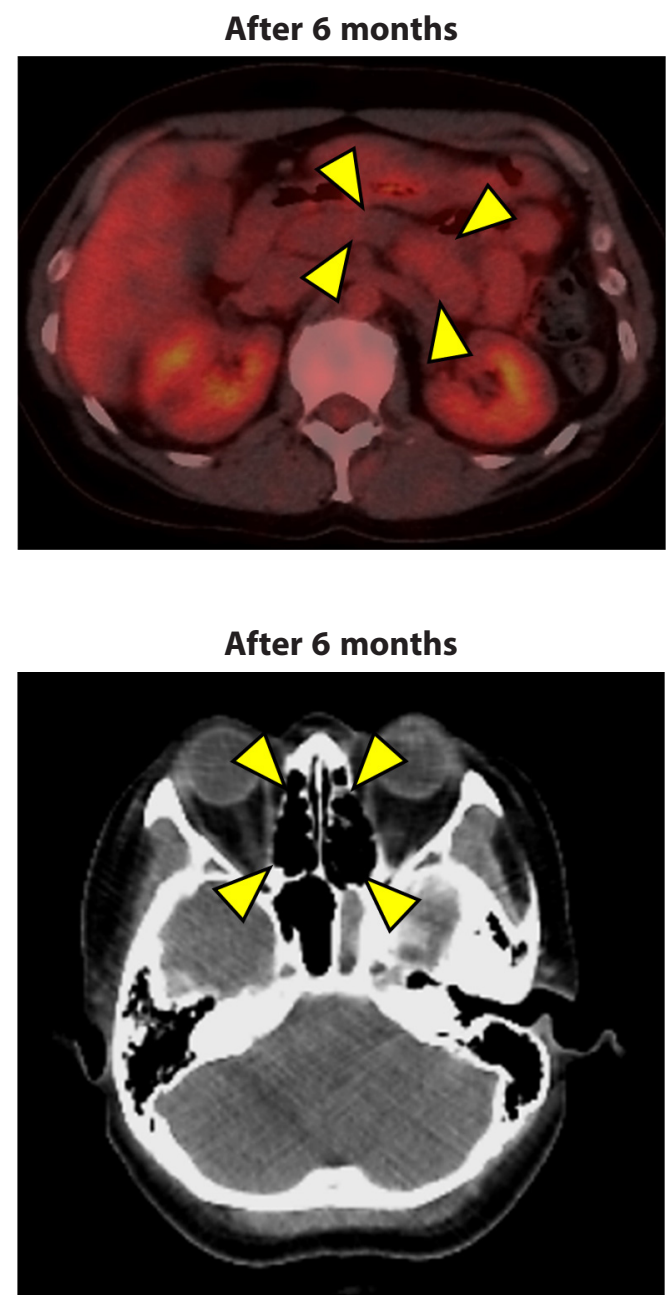

Figure 1.

(a) ${ }^{18}$ F-fluorodeoxyglucose positron emission tomography (FDG-PET) of the pancreas before and after prednisolone (PSL) treatment. Remarkable uptake of FDG was seen in the enlarged pancreas before treatment (left, arrowheads) and completely disappeared after treatment (right, arrowheads).

(b) Computed tomography of the ethmoid sinus before and after PSL treatment. Severe nasal mucosa hypertrophy in the bilateral ethmoid sinus before treatment (left, arrowheads) completely disappeared after treatment (right, arrowheads). 
C

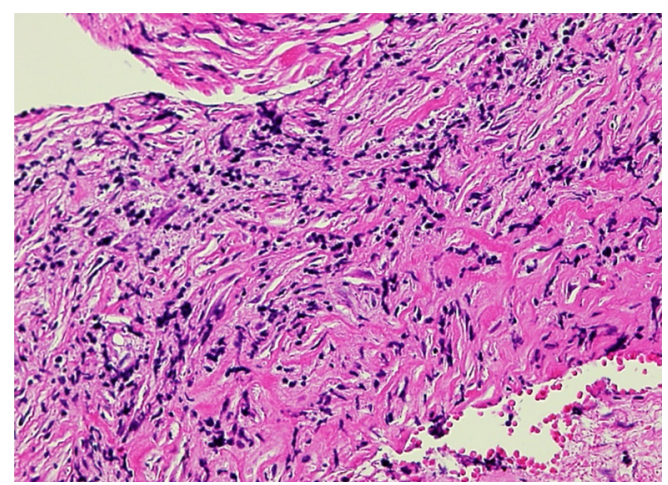

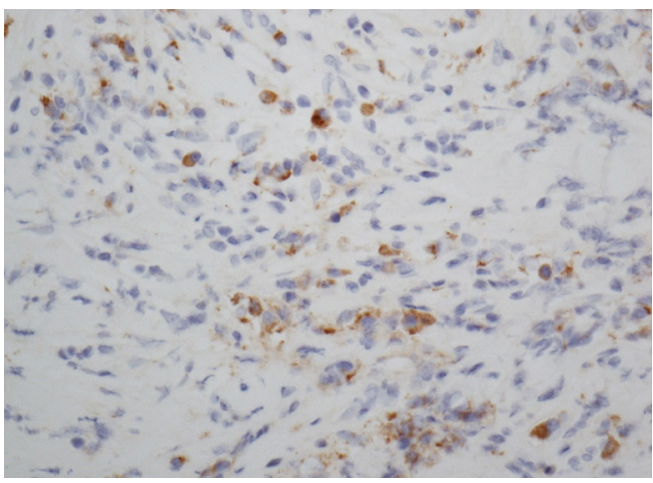

Figure 1. (Continued)

(c) Pathological examination of the pancreas tissue obtained by endoscopic ultrasound-guided fine needle aspiration. Formation of storiform fibrosis was observed in the Hematoxylin and Eosin staining (left). Infiltration of IgG4-expressing plasmacytes were observed in IgG4 immunostaining (right).

characteristic findings of type 1 AIP/IgG4-RD. These findings led us to diagnose this case as concurrent type 1 AIP/IgG4RD and CRS. ${ }^{1,2}$

She was treated with prednisolone (PSL) $35 \mathrm{mg}$, with a tapering schedule of $5 \mathrm{mg}$ every two weeks. No other treatment such as antibiotics or nasal irrigation has been applied. Six months after the initiation of PSL treatment, FDG uptake in the pancreas, salivary glands, and lacrimal glands completely disappeared (Figure 1a, right). Moreover, there was resolution of the CRS-associated symptoms, such as headache, nasal discharge, and stuffy nose. Follow-up CT after PSL treatment showed resolution of mucosal hypertrophy in the ethmoid sinus (Figure 1b, right).

The patient's course demonstrated that complete remission of both type 1 AIP/IgG4-RD and CRS was successfully induced by PSL treatment; this prompted us to examine the molecular mechanisms of both conditions by comparing the serum concentrations of total IgG, IgG1, IgG2, IgG3, and IgG4 before and after PSL treatment. The serum concentrations of IgG1, IgG2, and IgG3 were determined by enzyme-linked immuno-sorbent assay (ELISA) kits as previously described ${ }^{8}$

a

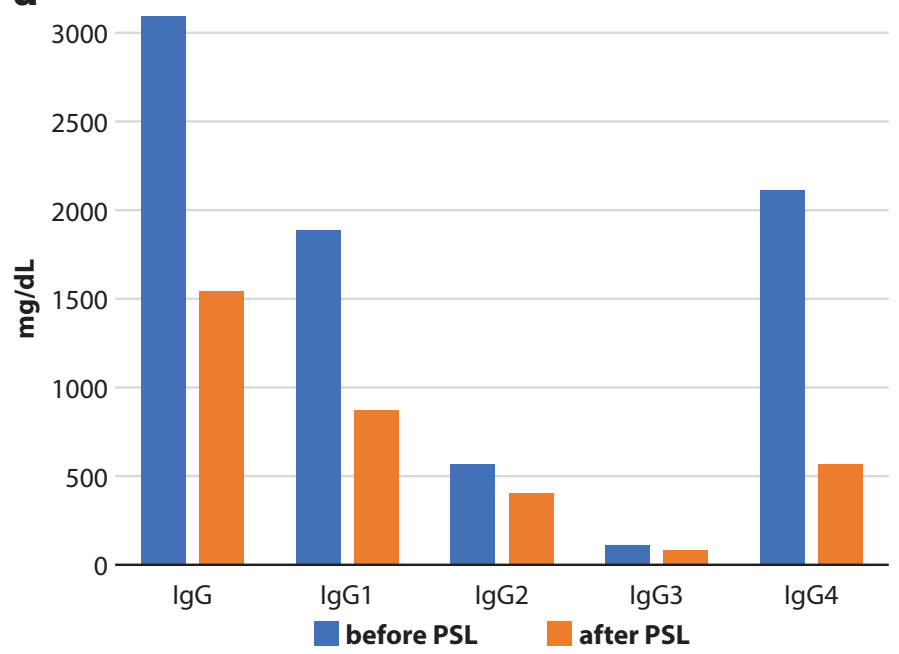

(Thermo Fisher Scientific, Waltham, MA). Additionally, using ELISA kits, we measured the serum concentrations of IL$1 \beta$ and IL-6 (Thermo Fisher Scientific) and IFN- $\alpha$ and IL-33 (R\&D systems, Minneapolis, MN) as previously described. ${ }^{8}$ After PSL treatment, there was marked decrease in the serum concentrations of total $\operatorname{IgG}, \operatorname{IgG} 1$, and $\operatorname{IgG} 4$ but not of $\operatorname{IgG} 2$ and IgG3 (Figure 2a). We previously reported that pDCs, which produce both IFN- $\alpha$ and IL-33, mediated murine experimental AIP and human IgG4-RD. Indeed, accumulation of pDCs in the pancreas and significant elevations of serum IFN- $\alpha$ and IL-33 concentrations were observed in patients with type 1 AIP, which is a pancreatic manifestation of systemic IgG4-RD. ${ }^{3-7}$ Interestingly, marked reduction in the serum concentrations of IFN- $\alpha$ and IL-33 was seen after induction of remission by PSL, whereas the concentrations of IL- $1 \beta$ and IL- 6 were comparable before and after PSL treatment (Figure $\mathbf{2 b}$, and data not shown). These data strongly suggested that the suppression of IFN- $\alpha$ and IL-33 production in this present case was associated with the remission of IgG4-RD and CRS.

b

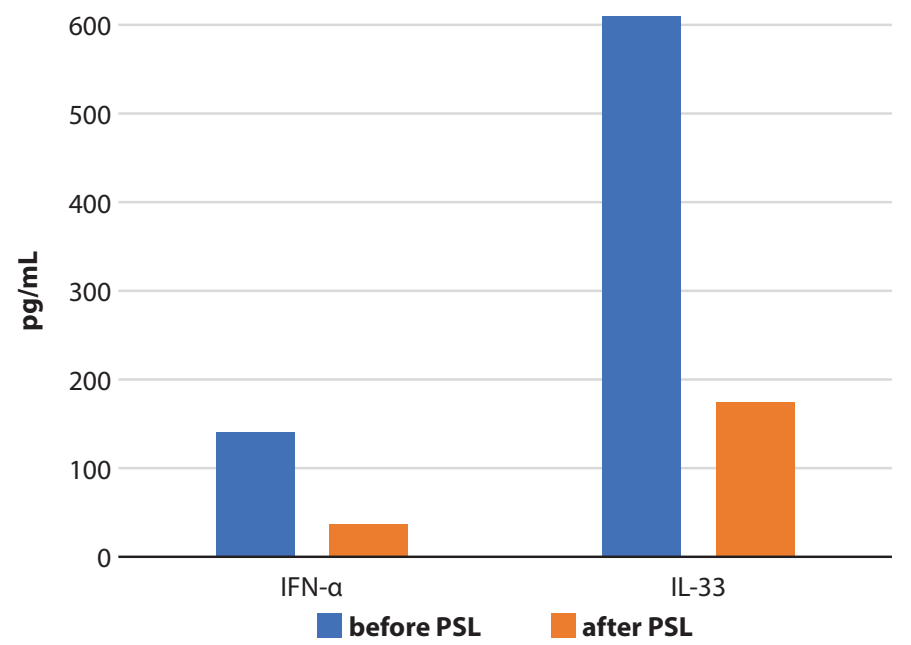

Figure 2. (a) Serum concentrations of total IgG, IgG1, IgG2, IgG3, and IgG4 before and after prednisolone (PSL) treatment. (b) Serum concentrations of IFN- $\alpha$ and IL-33 before and after PSL treatment. 


\section{Discussion}

Here we report a case of a patient with concurrent type 1 AIP/IgG4-RD and CRS. This case exhibited typical serological and pathological findings of type 1 AIP and IgG4-RD. The presence of CRS was confirmed by her symptoms and typical CT findings. Interestingly, PSL treatment markedly improved both type 1 AIP/IgG4-RD and CRS, which was confirmed by FDG-PET and CT after treatment.

Our analyses of serum cytokines suggested the possible involvement of IFN- $\alpha$ and IL-33 responses in the development of not only IgG4-RD but also CRS. As mentioned above, the development of murine experimental AIP and human IgG4$\mathrm{RD}$ partially depended on pDCs activation, followed by the production of IFN- $\alpha$ and IL-33. ${ }^{3-7}$ In line with this idea, serum concentrations of IFN- $\alpha$ and IL-33 are useful for the diagnosis and evaluation of disease activity in type 1 AIP/IgG4-RD as shown in our recent study. ${ }^{8}$ In terms of pathophysiology of CRS, previous studies reported that patients with CRS had increased expression of IL-33 in the nasal mucosa, although those did not examine the involvement of IFN- $\alpha^{13,14}$ Those previous studies and this present study strongly suggested that enhanced IL-33 production might be the prominent feature of both IgG4-RD and CRS and that PSL might induce the remission of both disorders through down-regulation of IL-33 responses.

One question that may arise from this case is whether CRS developed as a nasal mucosal manifestation of systemic IgG4-RD. CRS is classified into the noneosinophilic and eosinophilic types; the latter had been associated with a recurrent or refractory phenotype. ${ }^{15}$ According to the Japanese Epidemiological Survey of Refractory Eosinophilic Chronic Rhinosinusitis diagnostic system which suggests the diagnosis is eosinophilic chronic rhinosinusitis when the score is $\geq 11$, her score was $9 .{ }^{15}$ From the score criteria, her diagnosis was a non-eosinophilic chronic rhinosinusitis. Gao et al reported a high $(30 / 46,65.2 \%)$ incidence of CRS in patients with IgG4-RD and found that eye involvement in IgG4-RD was associated with CRS. ${ }^{11}$ Given the fact that the present case had lacrimal lesions and that the serum IL-33 responses declined after PSL treatment, which was similar to previously reported IgG4-RD cases, her CRS was likely a nasal mucosal manifestation of IgG4-RD. However, pathologic examination of nasal mucosal biopsy specimens would be required to confirm that CRS was a nasal mucosal manifestation of IgG4-RD in this case.

Finally, we encountered a case of IgG4-RD and CRS that were both successfully treated with PSL. Induction of remission by PSL was accompanied by marked reductions in the serum concentrations of IgG4, IFN- $a$, and IL-33. Given the fact that IL-33 was a common pathogenic cytokine of IgG4-RD and CRS, this case report strongly suggested that these two disorders can occur concurrently. In such cases, PSL treatment may be effective for both disorders. In addition, measurement of serum IgG4, which is a biomarker of postoperative recurrence of CRS, ${ }^{10}$ might be useful to determine the prognosis of the subpopulation of CRS that responds well to PSL. Further studies on a large number of patients with IgG4$\mathrm{RD}$ and CRS are absolutely required to confirm this idea.

\section{Conflict of interest}

Tomoe Yoshikawa, Kosuke Minaga, Akane Hara, Ikue Sekai, Yasuo Otsuka, Ryutaro Takada, Ken Kamata, Tomohiro Watanabe and Masatoshi Kudo declare that they have no conflict of interest.

\section{Human rights}

All procedures followed have been performed in accordance with the ethical standards laid down in the 1964 Declaration of Helsinki and its later amendments.

\section{Informed consent}

Informed consent was obtained from a patient for being included in the study.

\section{References}

1. Kamisawa T, Zen Y, Pillai S, Stone JH. IgG4-related disease. Lancet. 2015;385:1460-71.

2. Watanabe T, Minaga K, Kamata K, Kudo M, Strober W. Mechanistic Insights into Autoimmune Pancreatitis and IgG4-Related Disease. Trends Immunol. 2018;39:874-89.

3. Arai Y, Yamashita K, Kuriyama K, Shiokawa M, Kodama Y, Sakurai T, et al. Plasmacytoid Dendritic Cell Activation and IFN-alpha Production Are Prominent Features of Murine Autoimmune Pancreatitis and Human IgG4-Related Autoimmune Pancreatitis. J Immunol. 2015;195:3033-44.

4. Watanabe T, Yamashita K, Arai Y, Minaga K, Kamata K, Nagai T, et al. Chronic Fibro-Inflammatory Responses in Autoimmune Pancreatitis Depend on IFN-alpha and IL-33 Produced by Plasmacytoid Dendritic Cells. J Immunol. 2017;198:3886-96.

5. Kamata K, Watanabe T, Minaga K, Hara A, Yoshikawa T, Okamoto A, et al. Intestinal dysbiosis mediates experimental autoimmune pancreatitis via activation of plasmacytoid dendritic cells. Int Immunol. 2019;31:795-809.

6. Yoshikawa T, Watanabe T, Minaga K, Kamata K, Kudo M. Cytokines produced by innate immune cells in IgG4-related disease. Mod Rheumatol. 2019;29:219-25.

7. Minaga K, Watanabe T, Arai Y, Shiokawa M, Hara A, Yoshikawa T, et al. Activation of interferon regulatory factor 7 in plasmacytoid dendritic cells promotes experimental autoimmune pancreatitis. J Gastroenterol. 2020;55:565-76.

8. Minaga K, Watanabe T, Hara A, Kamata K, Omoto S, Nakai A, et al. Identification of serum IFN-alpha and IL-33 as novel biomarkers for type 1 autoimmune pancreatitis and IgG4-related disease. Sci Rep. 2020;10: 14879 .

9. Furukawa S, Moriyama M, Miyake K, Nakashima H, Tanaka A, Maehara $\mathrm{T}$, et al. Interleukin-33 produced by M2 macrophages and other immune cells contributes to Th2 immune reaction of IgG4-related disease. Sci Rep. 2017;7:42413.

10. Oka A, Ninomiya T, Fujiwara T, Takao S, Sato Y, Gion Y, et al. Serum IgG4 as a biomarker reflecting pathophysiology and post-operative recurrence in chronic rhinosinusitis. Allergol Int. 2020;69:417-23.

11. Gao Y, Zheng M, Cui L, Chen N, Wang YN, Zhan YT, et al. IgG4-related disease: association between chronic rhino-sinusitis and systemic symptoms. Eur Arch Otorhinolaryngol. 2018;275:2013-9.

12. Mafee MF, Tran BH, Chapa AR. Imaging of rhinosinusitis and its complications: plain film, CT, and MRI. Clin Rev Allergy Immunol. 2006;30:165-86.

13. Luo X, Li C, Wang Y, Yang G, Xu Y, Li G, et al. Interleukin-33 Promotes Th2/Th17 Response in Eosinophilic and Non-Eosinophilic Nasal Polyps. ORL J Otorhinolaryngol Relat Spec. 2020;82:34-9.

14. Drake LY, Kita H. IL-33: biological properties, functions, and roles in airway disease. Immunol Rev. 2017;278:173-84.

15. Tokunaga T, Sakashita M, Haruna T, Asaka D, Takeno S, Ikeda H, et al. Novel scoring system and algorithm for classifying chronic rhinosinusitis: the JESREC Study. Allergy. 2015;70:995-1003. 